\title{
Chemerin/ChemR23 signaling mediates the effects of ultra-high molecular weight polyethylene wear particles on the balance between osteoblast and osteoclast differentiation
}

\author{
Fengchao Zhao ${ }^{1}$, Dingwei Cang ${ }^{2}$, Jianzhi Zhang $^{3}$, Li Zheng ${ }^{4}$ \\ ${ }^{1}$ Department of Orthopedic Surgery, the First Affiliated Hospital, Zhejiang University, Hangzhou, China; ${ }^{2}$ Department of Orthopedic Surgery, \\ the First People's Hospital of Yancheng City, Yancheng, China; ${ }^{3}$ Department of Central Laboratory, the Affiliated Hospital of Xuzhou Medical \\ University, Xuzhou, China; ${ }^{4}$ Department of Orthopedic Surgery, the Affiliated Hospital of Xuzhou Medical University, Xuzhou, China \\ Contributions: (I) Conception and design: D Cang; (II) Administrative support: None; (III) Provision of study materials or patients: D Cang; (IV) \\ Collection and assembly of data: F Zhao, J Zhang, L Zheng; (V) Data analysis and interpretation: F Zhao, D Cang; (VI) Manuscript writing: All \\ authors; (VII) Final approval of manuscript: All authors. \\ Correspondence to: Dingwei Cang. Department of Orthopedic Surgery, the First People's Hospital of Yancheng City, 16 Yuehe Road, Yancheng \\ 224000, China. Email: 358752169@qq.com.
}

\begin{abstract}
Background: Ultra-high molecular weight polyethylene (UHMWPE) is one of the favored materials for total joint replacement, but its wear particles cause osteolysis. This study aims to elucidate the signaling that mediates the effects of UHMWPE particles on bone cells.

Methods: RAW264.7 and MC3T3-E1 cells were treated with UHMWPE particles. Chemerin/ChemR23 signaling was manipulated by either overexpressing Rarres 2 and Cmklr1 or silencing Cmklr1. The osteoblast and osteoclast differentiation was evaluated by Alizarin red and TRAP staining, respectively. The expression of osteogenic and osteoclastogenic markers was assessed with quantitative real time PCR and western blot.

Results: UHMWPE particles upregulated the expression of Rarres 2 and Cmklr1 in both osteoblast and osteoclast precursor cells. UHMWPE particles induced osteoclast differentiation while inhibited osteoblast differentiation, and this effect was abrogated by silencing Cmklr1 but augmented by the overexpression of Rarres2 and Cmklr1. Similarly, the expression of osteogenic marker genes was inhibited while that of osteoclastogenic marker genes was activated by UHMWPE particles, and this effect was abolished by silencing Cmklr1 and enhanced by Rarres 2 and Cmklr1 overexpression.

Conclusions: These results demonstrated that chemerin/ChemR23 signaling plays a central role in the effects of UHMWPE particles on the balance of osteogenic and osteoclastogenic differentiation, which changes the course of bone remodeling and eventually results in osteolysis.
\end{abstract}

Keywords: Ultra-high molecular weight polyethylene wear particle (UHMWPE wear particle); chemerin; ChemR23; osteoblast differentiation; osteoclast differentiation

Submitted Apr 27, 2021. Accepted for publication Jul 15, 2021.

doi: 10.21037/atm-21-2945

View this article at: https://dx.doi.org/10.21037/atm-21-2945

\section{Introduction}

Ultra-high molecular weight polyethylene (UHMWPE) has long been the material of choice for the load bearing in total joint replacements (e.g., the tibial plateau in total knee replacements and the acetabular cup in total hip prostheses) due to its excellent energetic toughness and inherent biocompatibility (1). Even with numerous improvements, oxidative damage and wear remain the main and interconnected material-related causes of UHMWPE failure, as oxidative degradation leads to decreased wear resistance $(1,2)$. UHMWPE wear particles cause the two closely connected processes of inflammation and 
osteolysis (3-5). Wear particles elicit the production of proinflammatory cytokines and chemokines $(5,6)$ that recruit and polarize macrophages and osteoclast precursor cells in periprosthetic tissues, increase local osteoclastogenesis, and inhibit osteoblast differentiation and function (7-9), which in turn leads to prosthesis loosening. However, it is not yet evident which signaling pathway functions as the master mediator of UHMWPE particles in disrupting the balance between osteogenesis and osteoclastogenesis.

Chemerin is widely considered an adipokine as it is highly expressed in adipocytes and plays autocrine and paracrine roles in adipose development. It also plays an endocrine role by modulating immunity and metabolism (10). Chemerin binds the G-protein-coupled receptors Cmklr1 (known as ChemR23) and GPR1 to activate a variety of downstream signaling pathways $(11,12)$. ChemR23 is widely expressed in immune cells and allows quick recruitment and activation of these immune cells upon chemerin signaling (11). Chemerin/ ChemR23 signaling promotes joint inflammation (13) and plays a role in molar tooth development (14). Silencing either chemerin or ChemR23 in bone marrow stem cells inhibits adipocyte differentiation but enhances osteoblast differentiation and mineralization (15). On the other hand, neutralization of chemerin blocks the osteoclast differentiation of hematopoietic stem cells, and the reintroduction of exogenous chemerin can reverse this effect (16). The aim of this study is to examine the role of chemerin/ChemR23 signaling in UHMWPE particleinduced osteolysis. We present the following article in accordance with the MDAR reporting checklist (available at https://dx.doi.org/10.21037/atm-21-2945).

\section{Methods}

\section{Cell culture and transfection}

MC3T3-E1 and RAW264.7 cells were purchased from the cell bank of the Chinese Academy of Sciences (Shanghai, China). MC3T3-E1 was maintained in $\alpha$-MEM and RAW264.7 in DMEM (high glucose), respectively, supplemented with $10 \% \mathrm{FBS}, 100 \mathrm{U} / \mathrm{mL}$ penicillin, and $100 \mathrm{mg} / \mathrm{mL}$ streptomycin (all from Gibco, Shanghai, China) at $37^{\circ} \mathrm{C}$ in a humidified atmosphere with $5 \% \mathrm{CO}_{2}$.

Runx2, chemerin, and ChemR23 overexpression and NfatC1, chemerin, and ChemR23 shRNA knockdown plasmids were synthesized and cloned by GenScript (Nanjing, China). Transfection was achieved with lipofectamine 3000 (ThermoFisher, Shanghai, China) according to the manufacturer's protocols.

\section{Osteoblast differentiation}

After reaching $80 \%$ confluency, MC3T3-E1 cells were changed to an osteoblast differentiation medium (a complete $\alpha$-MEM culture medium containing $10 \mathrm{mmol} / \mathrm{L} \beta$-glycerol phosphate (G9422, Sigma-Aldrich, Shanghai, China), $50 \mathrm{mg} / \mathrm{L} \mathrm{L}$-Ascorbic acid (A4544, Sigma-Aldrich), and $10 \mathrm{mmol} / \mathrm{L}$ dexamethasone (D4902, Sigma-Aldrich) for 3 weeks. The differentiation medium was changed every 3 days.

\section{Induction of osteoclast differentiation with UHMWPE particles}

We seeded $2 \times 10^{4}$ RAW264.7 cells into a 96-well plate and cultured them to $80 \%$ confluency. Then, $10^{8}$ UHMWPE particles (17) were added into each well. The culture medium was changed every $48 \mathrm{~h}$. The induction lasted for 7 days.

\section{Alizarin red staining}

The medium was removed. The cells were washed once with $\mathrm{PBS}$ and fixed in $70 \%$ ethanol at room temperature for $30 \mathrm{~min}$. The cells were washed three times with PBS and incubated with Alizarin red S staining solution (Solarbio, Beijing, China) at $37{ }^{\circ} \mathrm{C}$ for $30 \mathrm{~min}$. The cells were washed twice with $\mathrm{ddH}_{2} \mathrm{O}$ before being examined and photographed using XDS-1A microscopy (Wuzhou New Found Instrument, Wuzhou, China).

\section{TRAP staining}

The cells were spun at $500 \mathrm{~g}$ for $5 \mathrm{~min}$, washed twice with PBS, fixed with $70 \%$ ethanol at room temperature for $30 \mathrm{~min}$, and stained with a Leukocyte Acid Phosphatase Kit (387A, Sigma, St. Louis, MO, USA). TRAP-positive cells with three or more nuclei were counted as mature osteoclasts.

\section{Quantitative real-time PCR}

Total RNA was extracted from the cells using an RNA purification kit (DP412, Tiangen Bio, Beijing, China) and reverse transcribed using a TIANScript RT Kit (KR107, Tiangen Bio). Quantitative real-time PCR was performed with an AB 7500 system (Applied Biosystems, Forest City, CA, USA) using a Talent fluorescence quantitative detection kit (SYBR Green) (FP209, Tiangen Bio). The primers used in this study are listed in Table 1. Relative gene expression was calculated with the $2^{-\Delta \Delta \mathrm{Ct}}$ method with GAPDH as the internal control. 
Table 1 Sequences of primers used in this study

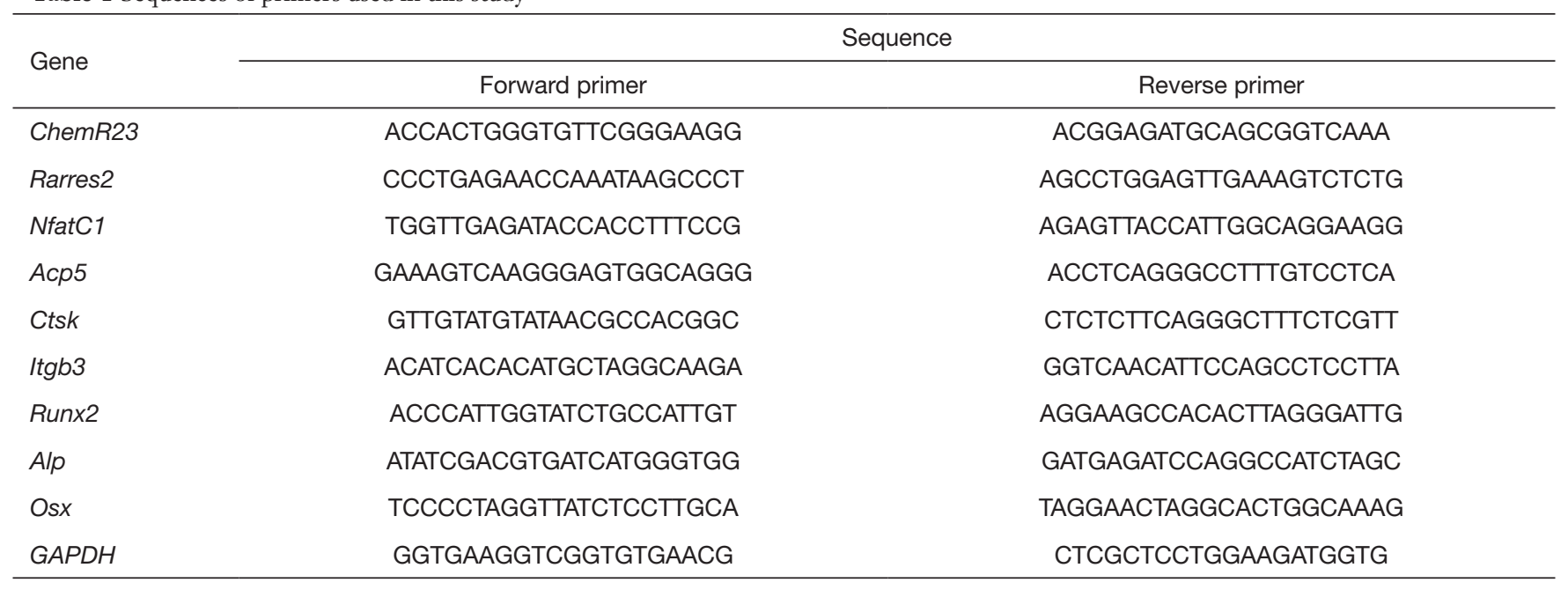

\section{Western blot}

The cells were lysed on ice for 10 min with RIPA lysis buffer (P0013C, Beyotime, Shanghai, China) containing 1× Protease Phosphatase Inhibitor Cocktail (P1050, Beyotime) before being centrifuged at $13,000 \mathrm{~g}$ for $10 \mathrm{~min}$ at $4{ }^{\circ} \mathrm{C}$. Total protein samples $(40 \mu \mathrm{g})$ were resolved on $8 \%$ SDS-PAGE gel and transferred onto PVDF membranes. The membranes were blocked with $5 \%$ nonfat milk in PBST $(0.05 \%$ Tween 100 in PBS), incubated with specified primary antibodies overnight at $4{ }^{\circ} \mathrm{C}$, washed and incubated with a horseradish peroxidase-conjugated secondary antibody (Jackson ImmunoResearch, West Grove, PA, USA), and then washed and detected with an enhanced chemiluminescence substrate (Roche, Indianapolis, IN, USA).

\section{Statistical analysis}

All experiments were independently performed three times with triplicates. Data are shown as mean \pm standard deviation of the mean. All statistical analyses were performed using Prism 5 (Graphpad, San Diego, CA, USA). The differences between groups were analyzed by one-way analysis of variance followed by the Bonferonni test. A P value $<0.05$ was considered statistically significant.

\section{Results}

UHMWPE wear particles upregulated chemerin and ChemR23 expression

As the chemerin/ChemR23 system is known to play an important role in both osteoblastogenesis and osteoclastogenesis $(15,16)$, we first investigated whether UHMWPE wear particles regulated the expression of chemerin (Rarres2) and ChemR23 in bone cells. The UHMWPE particles gradually upregulated Rarres 2 (Figure 1A) and ChemR23 (Figure 1B) during both osteoclast (Figure $1 A, B$ ) and osteoblast (Figure 1C,D) differentiation.

\section{Chemerin/ChemR23 mediated UHMWPE wear particle- induced osteoclastogenesis}

As UHMWPE particles were previously shown to induce osteoclast differentiation (17) and osteoclastogenesis was blocked by chemerin neutralization (16), we examined the role of chemerin/ChemR23 in UHMWPE particle-induced osteoclast differentiation. We found that UHMWPE particles induced RAW264.7 macrophages to differentiate into osteoclasts (Figure 2A). Silencing ChemR23 expression alone did not change the behavior of the RAW264.7 cells but greatly inhibited UHMWPE particle-induced osteoclast differentiation. Overexpression of chemerin and ChemR23 caused the RAW264.7 cells to spontaneously differentiate into osteoclasts and enhanced the osteoclastogenic effect of the UHMWPE particles (Figure 2A).

The mRNA (Figure 2B) and protein (Figure 2C) levels of the osteoclastogenic gene $\mathrm{NfatCl}$ and the osteoclast markers Itgb3, Acp 5, and CtsK were increased by about 2- to 4-fold by the UHMWPE particles, and this effect was almost completely abrogated by ChemR23 silencing. Overexpressing chemerin and ChemR23 upregulated the NfatC1, Itgb3, Acp5, and $C t s K$ expression and further augmented the effects of the 

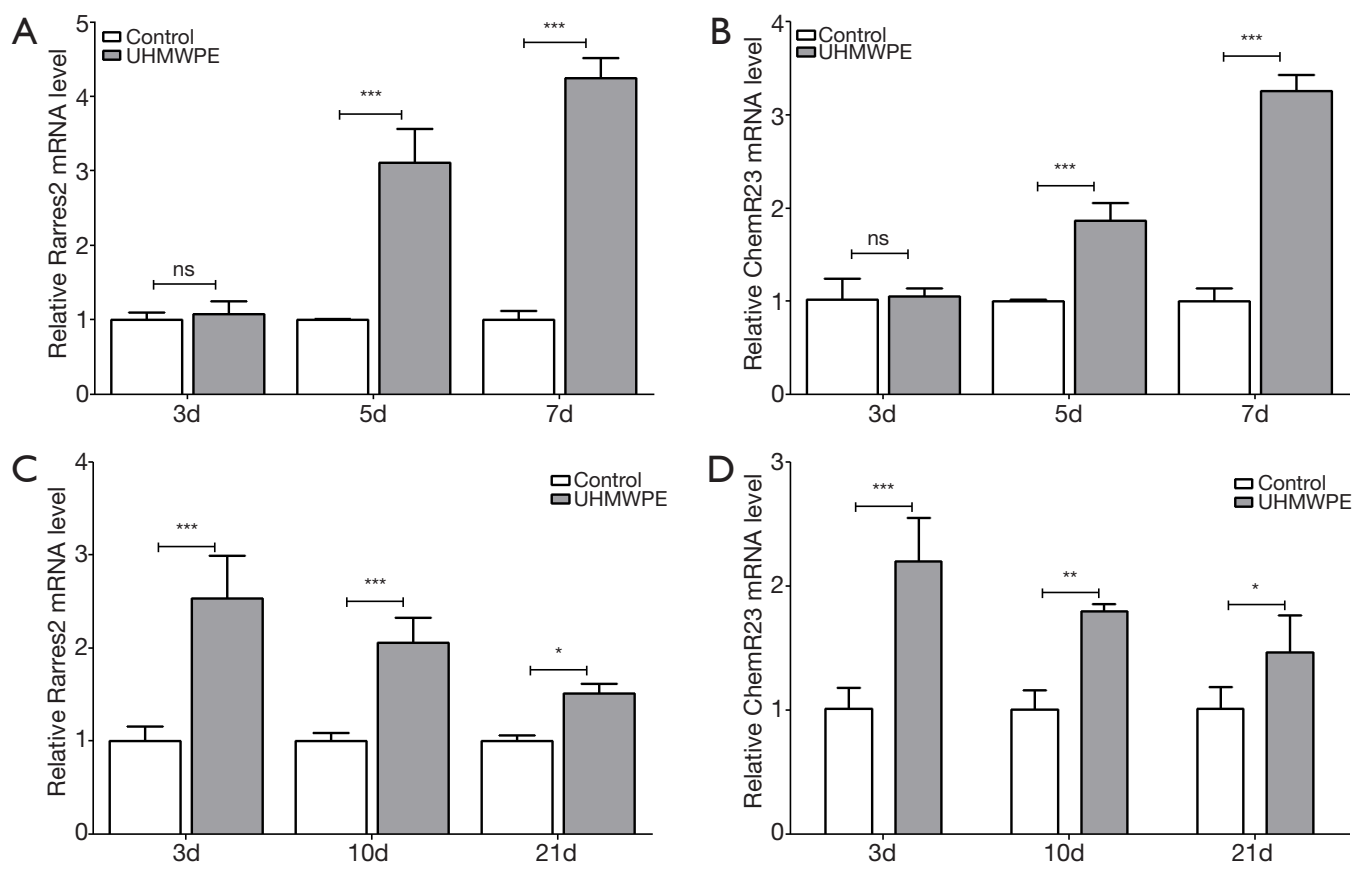

Figure 1 UHMWPE particles upregulate Rarres2 and ChemR23 expression during osteoblast and osteoclast differentiation. RAW264.7 cells are treated with UHMWPE particles for 3, 5, and 7 days (A,B). MC3T3-E1 cells are cultured in an osteogenic medium with or without UHMWPE particles for 3, 10, and 21 days (C,D), and the Rarres2 (A,C) and ChemR23 (B,D) mRNA levels are analyzed by quantitative realtime PCR. *, $\mathrm{P}<0.05$; **, $\mathrm{P}<0.01$; **, $\mathrm{P}<0.001$. UHMWPE, ultra-high molecular weight polyethylene.

UHMWPE particles (Figure 2B,C).

\section{AP1 and NF- $\kappa B$ were activated by UHMWPE particles in RAW264.7 cells}

We then looked into the activation of $A P-1$ and $N F-\kappa B$ in RAW264.7 cells upon exposure to UHMWPE particles with or without intervening chemerin signaling. Neither $c$-fos nor $N F-\kappa B$ p 65 was phosphorylated 15 seconds after UHMWPE particle treatment (Figure $3 A$ ). However, the levels of phosphorylated $c$-fos and $N F-\kappa B p 65$ were significantly increased after UHMWPE particle treatment for 45 (Figure 3B) and 120 (Figure 3C) seconds, and this effect was inhibited by shRNA silencing of ChemR23 and enhanced by chemerin and ChemR23 overexpression (Figure 3B,C).

\section{NfatC1 was required for chemerin/ChemR23-mediated induction of osteoclast differentiation by UHMWPE particles}

We next examined if $\mathrm{NfatC1}$ was essential for the UHMWPE particle induction of osteoclast differentiation as it is the master regulator of osteoclastogenesis (18). UHMWPE particles induced the overexpression of chemerin and ChemR23, upregulated osteoclastogenic genes $\mathrm{NfatC1}$, $A C P 5$, Ctsk, and Itgb3 (Figure 4A), and strongly induced the osteoclast differentiation of RAW264.7 cells (Figure 4B). Silencing $\mathrm{NfatC1}$ expression did not impact the UHMWPE particle-induced overexpression of chemerin and ChemR23 (Figure $4 A$ ) but inhibited the UHMWPE particle-induced expression of osteoclastogenic transcription factor NfatC1, the osteoclast specific markers Acp5, Ctsk, Itgb3 (Figure 4A), and osteoclast differentiation (Figure 4B).

\section{Silencing ChemR23 abrogated the inbibition of osteogenesis by UHMWPE particles}

UHMWPE particles inhibited osteoblast differentiation of MC3T3-E1 cells in a dose-dependent manner (Figure S1), and the expression of chemerin and ChemR23 was upregulated by UHMWPE particles (Figure 1C,D). We investigated the role of chemerin/ChemR23 signaling in the inhibition of osteoblast differentiation by UHMWPE particles. The UHMWPE particles significantly inhibited 

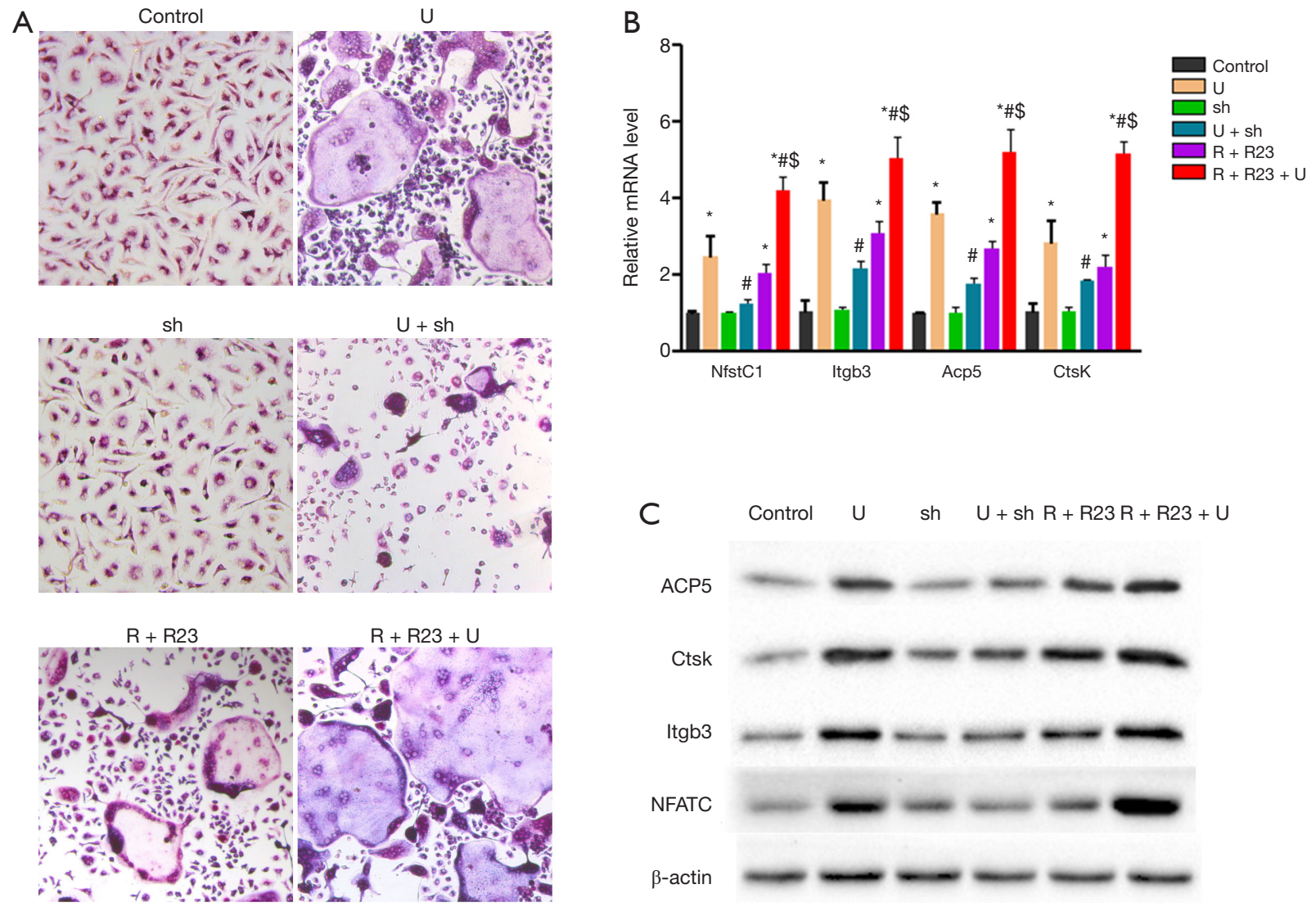

Figure 2 Chemerin/ChemR23 signaling mediates UHMWPE particle-induced osteoclast differentiation. (A) RAW264.7 cells are cultured in a complete medium with or without UHMWPE particles for 7 days and subjected to TRAP staining. (B) mRNA levels of osteoclastogenic markers NfatC1, Itgb3, Acp5, and Ctsk are examined by quantitative real-time PCR. (C) The protein levels of osteoclastogenic markers NfatC1, Itgb3, Acp5, and Ctsk are assessed by western blot. Bar $=100 \mu \mathrm{m}$. *, $\mathrm{P}<0.05$ compared to C; *, $\mathrm{P}<0.05$ compared to $\mathrm{U}$; ${ }^{\$}, \mathrm{P}<0.05$ compared to R + R23. UHMWPE, ultra-high molecular weight polyethylene; U, UHMWPE particles; sh, shRNA silencing Cmklr1 (ChemR23); R, Rarres2 (chemerin) overexpression; R23, Cmklr1 (ChemR23) overexpression.

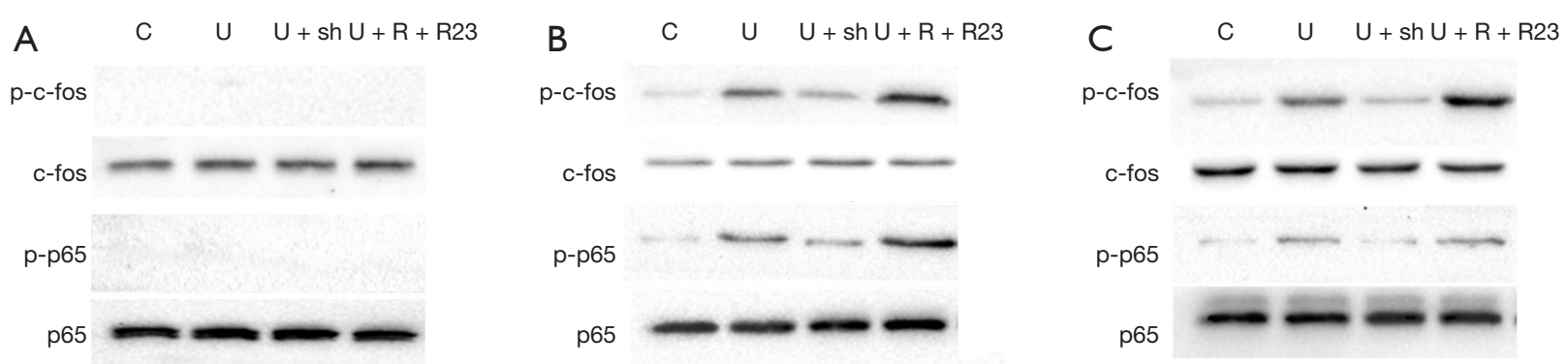

Figure 3 UHMWPE particles activate AP-1 and NF- $\mathrm{BB}$ signaling through Chemerin/ChemR23 system. RAW264.7 cells are transfected with shRNA targeting Cmklr1 or Rarres 2 and Cmklr1 overexpressing vectors (or their corresponding control vector). Forty-eight hours later, cells are exposed to UHMWPE particles for 15 (A), 45 (B), and 120 (C) seconds. The phosphorylation of c-fos and NF- $\kappa \mathrm{B}$ p65 is evaluated by western blot. UHMWPE, ultra-high molecular weight polyethylene; C, control; U, UHMWPE particles; sh, shRNA silencing Cmklr1 (ChemR23); R, Rarres2 (chemerin) overexpression; R23, Cmklr1 (ChemR23) overexpression. 
A

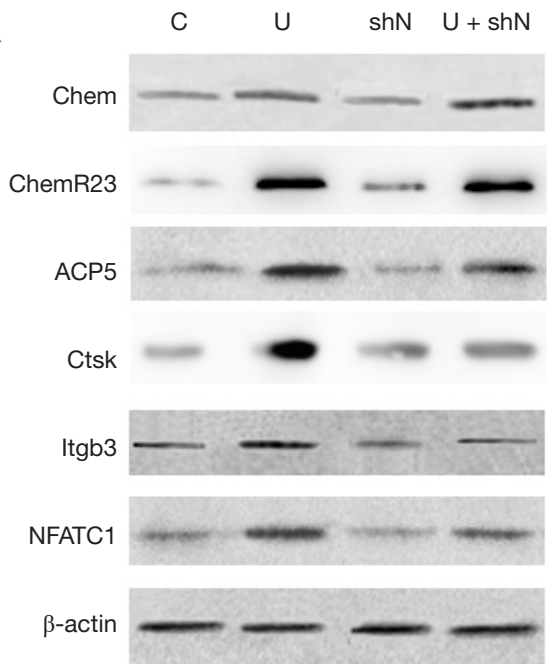

B

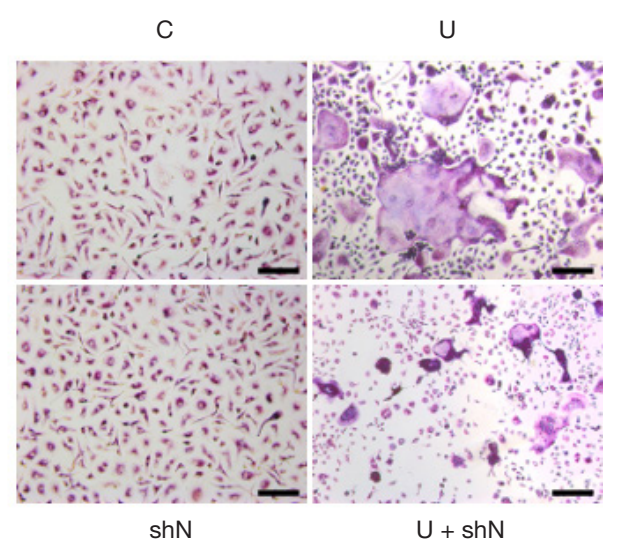

Figure 4 Chemerin/ChemR23 transduces the osteoclastogenic signal of UHMWPE particles through NfatC1. (A) The protein levels of chemerin, ChemR23, and the osteoclastogenic markers NfatC1, Itgb3, Acp5, and Ctsk are assessed by western blot. (B) RAW264.7 cells with or without $N f a t C 1$ silencing are cultured in complete medium with or without UHMWPE particles for 7 days and subjected to TRAP staining. Bar $=100 \mu \mathrm{m}$. UHMWPE, ultra-high molecular weight polyethylene; C, control; U, UHMWPE particles; shN, shRNA silencing NfatC1.

osteoblast differentiation (reduction of mineralized nodules). Silencing ChemR23 did not obviously impact the osteogenesis of the MC3T3-E1 cells. However, ChemR23 knockdown abolished the inhibition of the UHMWPE particles on osteoblast differentiation (Figure $5 A$ ).

Consistently, UHMWPE particles inhibited the mRNA (Figure 5B) and protein (Figure 5C) levels of the osteogenic genes Runx2, ALP, and Osx. Silencing ChemR23 relieved the inhibition of Runx2, ALP, and Osx expression by the UHMWPE particles (Figure $5 B, C$ ).

\section{RUNX1 transduced chemerin/ChemR23 signaling in mediating the inhibition of osteoblast differentiation by UHMWPE particles}

Overexpressing Runx2 did not change the levels of chemerin and ChemR23 in the MC3T3-E1 cells with or without UHMWPE particles (Figure $6 A$ ). However, ectopic Runx2 not only enhanced osteoblast differentiation of the MC3T3-E1 cells but also relieved the inhibition of osteoblast differentiation by the UHMWPE particles (Figure 6A,B).

\section{Discussion}

Bone health is maintained by the balance between osteoclasts and osteoblasts. Any disruption of this balance can lead to various diseases, including osteolysis, osteoporosis, and rheumatoid arthritis. For total joint replacement, periprosthetic osteolysis is the most significant long-term complication (19). Implant failure results from excessive bone resorption caused by prosthetic wear particles activating innate immune responses $(20,21)$. Activation of $N F-\kappa B$ plays a central role in osteoclastogenesis. UHMWPE particles induce the activation of $N F-\kappa B$ and promote the production of $T N F-\alpha$ and $T C P-1$, which leads to osteoclast differentiation (17). The current study showed that UHMWPE particles stimulated the phosphorylation of both $N F-\kappa B p 65$ and $c$-fos. Transcription factor $A P-1$ (c-fos and $c$-jun) and $N f a t C 1$ independently or cooperatively promoted the osteoclast cell marker gene cathepsin $\mathrm{K}$ (Ctsk) transcription (22). Inhibition of $N F-\kappa B$ and $A P-1$ activation suppressed the RANKL-induced osteoclast differentiation of RAW264.7 cells (23). These results are consistent with current data showing that UHMWPE particles activate $N F-\kappa B$ and $c$-fos (AP-1), upregulate osteoclastogenic genes, and induce osteoclast differentiation. Moreover, UHMWPE particles may induce the secretion of pro-osteoclastogenic factors by osteoblasts (24), which promotes bone resorption and eventually leads to osteolysis.

The differentiation, turnover, and function of osteoblasts is the other half of the equation in periprosthetic 

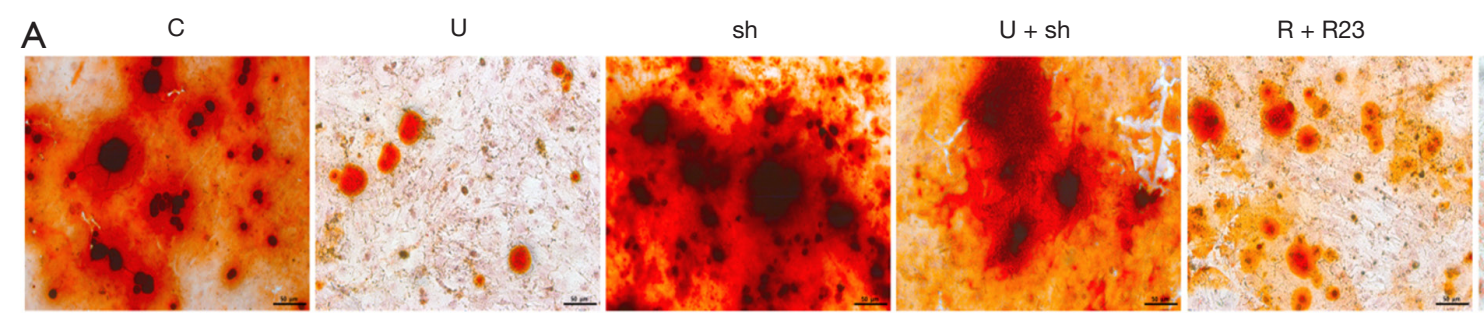

$\mathrm{U}+\mathrm{R}+\mathrm{R} 23$
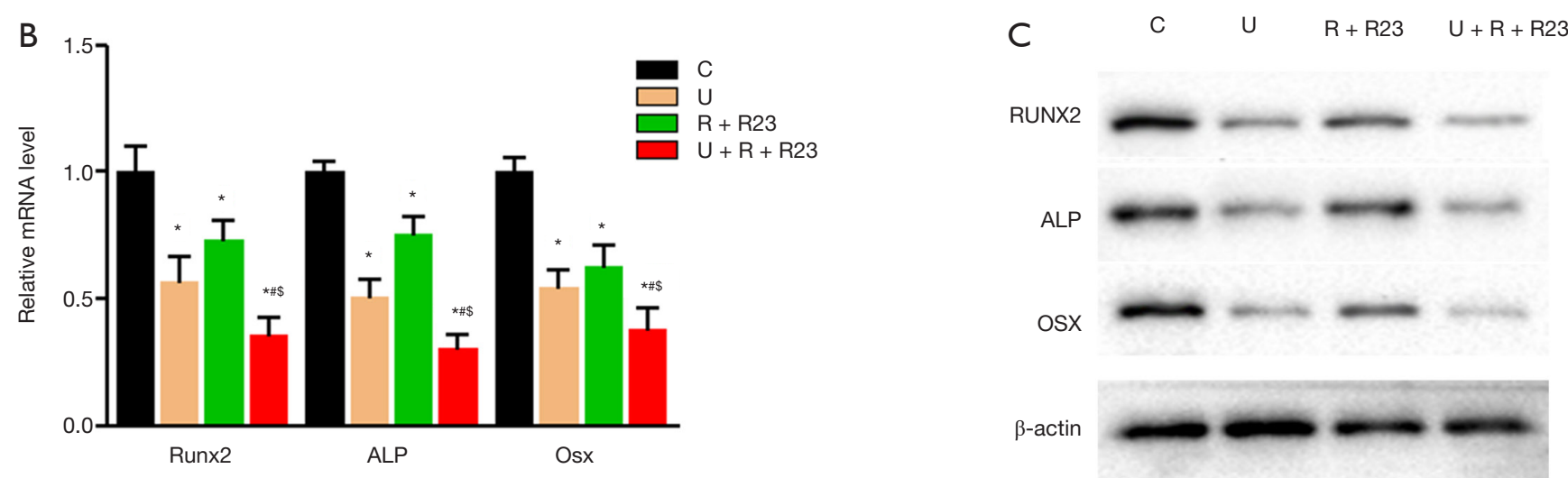

Figure 5 Chemerin/ChemR23 signaling is required for the inhibition of osteoblast differentiation by UHMWPE particles. MC3T3-E1 cells are transfected with shRNA targeting Cmklr1 or Rarres 2 and Cmklr1 overexpressing vectors (or their corresponding control vector). Forty-eight hours later, cells are cultured in an osteoblast differentiation medium with or without $4 \times 10^{6} / \mathrm{mL}$ UHMWPE particles for 21 days. (A) Mineral deposition is assessed with Alizarin red staining. (B) The mRNA levels of osteoblastogenic markers Runx2, Alp, and Osx are analyzed using quantitative real-time PCR. (C) The protein levels of osteogenic genes Runx2, Alp, and Osx are assayed by western blot. Bar $=50 \mu \mathrm{m}$. *, $\mathrm{P}<0.05$ compared to $\mathrm{C}$; ${ }^{*}, \mathrm{P}<0.05$ compared to $\mathrm{U} ;{ }^{\$}, \mathrm{P}<0.05$ compared to $\mathrm{R}+\mathrm{R} 23$. UHMWPE, ultra-high molecular weight polyethylene; C, control; U, UHMWPE particles; sh, shRNA silencing Cmklr1 (ChemR23); R, Rarres2 (chemerin) overexpression; R23, Cmklr1 (ChemR23) overexpression.

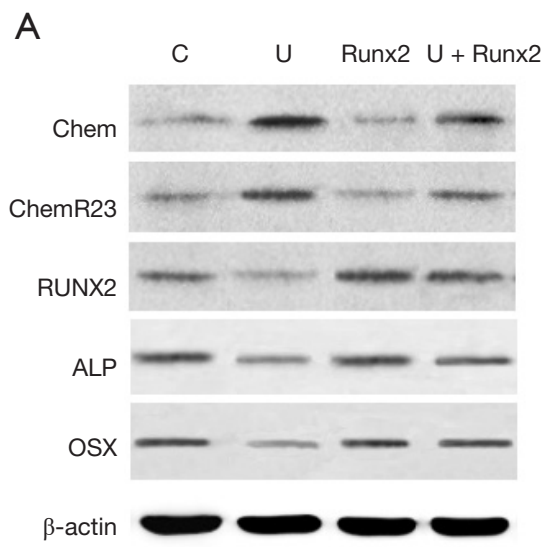

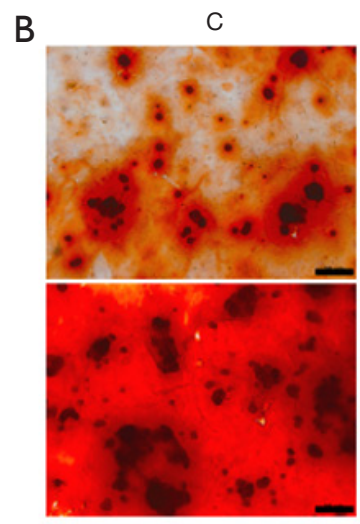

Runx2

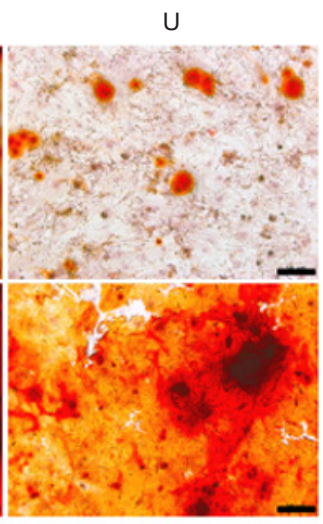

$\mathrm{U}+$ Runx2

Figure 6 Chemerin/ChemR23 signaling through Runx2 transduces the anti-osteogenic effect of UHMWPE particles. (A) The protein levels of chemerin, ChemR23, and the osteogenic markers Runx2, Alp, and Osx are assessed by western blot. (B) MC3T3-E1 cells are transfected with Runx2 overexpressing vectors (or their corresponding control vector). Forty-eight hours later, cells are cultured in an osteoblast induction medium with or without $4 \times 10^{6} / \mathrm{mL}$ UHMWPE particles for 21 days and subjected to Alizarin red staining. Bar $=100 \mu \mathrm{m}$. UHMWPE, ultra-high molecular weight polyethylene; C, control; U, UHMWPE particles; Runx2, Runx2 expression vector. 
osteolysis. Most studies have focused on osteoclasts, but prosthetic wear particles may impact osteoblasts and contribute to periprosthetic osteolysis by inhibiting osteoblast differentiation, proliferation, and activities, as well as stimulating osteoblasts to produce inflammatory cytokines (25). A previous study reported that $\mathrm{CoCrMo}$ metal particles induced apoptosis in MC3T3-E1 cells in vitro and caused particle-induced osteolysis by autophagy in the osteoblasts of mouse calvaria (26). The TSIX/miR$30 \mathrm{a}-5 \mathrm{p}$ axis was also shown to mediate CoCrMo metal particle-induced osteoblast apoptosis (27). Additionally, LncRNA DANCR inhibited osteoblast differentiation by downregulating the expressions of FOXO1, Runx2, Osterix $(O s t x)$, and osteocalcin $(O C N)$ in the osteolysis process after total hip arthroplasty (28). The current study demonstrated that UHMWPE particles dose-dependently inhibit osteoblast differentiation of MC3T3-E1 cells. Moreover, UHMWPE particles upregulate chemerin and ChemR23 expression in both osteoblast and osteoclast precursor cells.

Furthermore, we showed that chemerin/ChemR23 signaling plays different roles in mediating the UHMWPE particle effects on osteoblast and osteoclast differentiation. Inhibition of chemerin/ChemR23 signaling canceled out the inhibition of osteoblastogenesis by UHMWPE particles, which is consistent with the notion that chemerin/ ChemR23 is an anti-osteogenic signal $(15,16)$. Moreover, overexpression of Runx 2 abrogated the inhibitory effects of UHMWPE particles/chemerin/ChemR23 on osteoblast differentiation. Meanwhile, silencing ChemR23 expression abolished the osteoclastogenic effect of UHMWPE particles. Chemerin/ChemR23 has been reported to prompt $N F-\kappa B$ activation and inflammatory factors secretion (29). A further study reported that hematopoietic ChemR23 sustained the activated M1 macrophage population and promoted the homing of plasmacytoid dendritic cells to lymphatic organs and recruitment to atherosclerotic lesions (30). As chemerin can act as an autocrine, paracrine, and endocrine mediator, it may recruit monocytes/ macrophages to periprosthetic tissue, activate macrophages, and promote osteoclast differentiation upon exposure to UHMWPE wear particles.

\section{Conclusions}

Chemerin and ChemR23 were upregulated by UHMWPE wear particles in the precursor cells of both osteoblasts and osteoclasts. Silencing ChemR23 abolished the inhibitory effect on osteoblastogenesis and the inductive effect on osteoclastogenesis of the UHMWPE wear particles by regulating the master regulators $R u n \times 2$ in pre-osteoblasts and $\mathrm{NfatC1}$ in pre-osteoclasts. These results indicate that chemerin/ChemR23 signaling may be a novel target for preventing periprosthetic osteolysis.

\section{Acknowledgments}

Funding: This study was supported by the National Natural Science Foundation of China (No. 81672184) and the Primary Research \& Development Plan of Jiangsu Province (No. BE2016642).

\section{Footnote}

Reporting Checklist: The authors have completed the MDAR reporting checklist. Available at https://dx.doi. org/10.21037/atm-21-2945

Data Sharing Statement: Available at https://dx.doi. org/10.21037/atm-21-2945

Conflicts of Interest: All authors have completed the ICMJE uniform disclosure form (available at https://dx.doi. org/10.21037/atm-21-2945). The authors have no conflicts of interest to declare.

Ethical Statement: The authors are accountable for all aspects of the work in ensuring that questions related to the accuracy or integrity of any part of the work are appropriately investigated and resolved.

Open Access Statement: This is an Open Access article distributed in accordance with the Creative Commons Attribution-NonCommercial-NoDerivs 4.0 International License (CC BY-NC-ND 4.0), which permits the noncommercial replication and distribution of the article with the strict proviso that no changes or edits are made and the original work is properly cited (including links to both the formal publication through the relevant DOI and the license). See: https://creativecommons.org/licenses/by-nc-nd/4.0/.

\section{References}

1. Malito LG, Arevalo S, Kozak A, et al. Material properties of ultra-high molecular weight polyethylene: comparison of tension, compression, nanomechanics and 
microstructure across clinical formulations. J Mech Behav Biomed Mater 2018;83:9-19.

2. Ansari F, Ries MD, Pruitt L. Effect of processing, sterilization and crosslinking on UHMWPE fatigue fracture and fatigue wear mechanisms in joint arthroplasty. J Mech Behav Biomed Mater 2016;53:329-40.

3. Ren PG, Irani A, Huang Z, et al. Continuous infusion of UHMWPE particles induces increased bone macrophages and osteolysis. Clin Orthop Relat Res 2011;469:113-22.

4. Jiang J, Jia T, Gong W, et al. Macrophage polarization in IL-10 treatment of particle-induced inflammation and osteolysis. Am J Pathol 2016;186:57-66.

5. Jiang X, Sato T, Yao Z, et al. Local delivery of mutant CCL2 protein-reduced orthopaedic implant wear particleinduced osteolysis and inflammation in vivo. J Orthop Res 2016;34:58-64.

6. Yao Z, Keeney M, Lin TH, et al. Mutant monocyte chemoattractant protein 1 protein attenuates migration of and inflammatory cytokine release by macrophages exposed to orthopedic implant wear particles. J Biomed Mater Res A 2014;102:3291-7.

7. Shanbhag AS, Hasselman CT, Rubash HE. The John Charnley Award. Inhibition of wear debris mediated osteolysis in a canine total hip arthroplasty model. Clin Orthop Relat Res 1997;(344):33-43.

8. Harris WH. Wear and periprosthetic osteolysis: the problem. Clin Orthop Relat Res 2001;(393):66-70.

9. Kurcz B, Lyons J, Sayeed Z, et al. Osteolysis as it pertains to total hip arthroplasty. Orthop Clin North Am 2018;49:419-35.

10. Rourke JL, Dranse HJ, Sinal CJ. Towards an integrative approach to understanding the role of chemerin in human health and disease. Obes Rev 2013;14:245-62.

11. Helfer $\mathrm{G}, \mathrm{Wu}$ QF. Chemerin: a multifaceted adipokine involved in metabolic disorders. J Endocrinol 2018;238:R79-94.

12. Goralski KB, Jackson AE, McKeown BT, et al. More than an adipokine: the complex roles of chemerin signaling in cancer. Int J Mol Sci 2019;20:4778.

13. Berg V, Sveinbjörnsson B, Bendiksen S, et al. Human articular chondrocytes express ChemR23 and chemerin; ChemR23 promotes inflammatory signalling upon binding the ligand chemerin(21-157). Arthritis Res Ther 2010;12:R228.

14. Ohira T, Spear D, Azimi N, et al. ChemerinChemR23 signaling in tooth development. J Dent Res 2012;91:1147-53.

15. Muruganandan S, Roman AA, Sinal CJ. Role of
chemerin/CMKLR1 signaling in adipogenesis and osteoblastogenesis of bone marrow stem cells. J Bone Miner Res 2010;25:222-34.

16. Muruganandan S, Dranse HJ, Rourke JL, et al. Chemerin neutralization blocks hematopoietic stem cell osteoclastogenesis. Stem Cells 2013;31:2172-82.

17. Cang D, Guo K, Zhao F. Dendritic cells enhance UHMWPE wear particle-induced osteoclast differentiation of macrophages. J Biomed Mater Res A 2015;103:3349-54.

18. Asagiri M, Takayanagi H. The molecular understanding of osteoclast differentiation. Bone 2007;40:251-64.

19. Sultan AA, Samuel LT, Umpierrez E, et al. Routine use of commercial antibiotic-loaded bone cement in primary total joint arthroplasty: a critical analysis of the current evidence. Ann Transl Med 2019;7:73.

20. Cobelli N, Scharf B, Crisi GM, et al. Mediators of the inflammatory response to joint replacement devices. Nat Rev Rheumatol 2011;7:600-8.

21. Abu-Amer Y, Darwech I, Clohisy JC. Aseptic loosening of total joint replacements: mechanisms underlying osteolysis and potential therapies. Arthritis Res Ther 2007;9 Suppl 1:S6.

22. Pang M, Rodríguez-Gonzalez M, Hernandez M, et al. AP-1 and Mitf interact with NFATc1 to stimulate cathepsin $\mathrm{K}$ promoter activity in osteoclast precursors. J Cell Biochem 2019;120:12382-92.

23. Hwang YH, Lee JW, Hahm ER, et al. Momordin I, an inhibitor of AP-1, suppressed osteoclastogenesis through inhibition of NF-kappaB and AP-1 and also reduced osteoclast activity and survival. Biochem Biophys Res Commun 2005;337:815-23.

24. Di Pompo G, Errani C, Gillies R, et al. Acid-induced inflammatory cytokines in osteoblasts: a guided path to osteolysis in bone metastasis. Front Cell Dev Biol 2021;9:678532.

25. O'Neill SC, Queally JM, Devitt BM, et al. The role of osteoblasts in peri-prosthetic osteolysis. Bone Joint J 2013;95-B:1022-6.

26. Wang Z, Liu N, Liu K, et al. Autophagy mediated CoCrMo particle-induced peri-implant osteolysis by promoting osteoblast apoptosis. Autophagy 2015;11:2358-69.

27. Bu Y, Zheng D, Wang L, et al. LncRNA TSIX promotes osteoblast apoptosis in particle-induced osteolysis by down-regulating miR-30a-5p. Connect Tissue Res 2018;59:534-41.

28. Tang Z, Gong Z, Sun X. LncRNA DANCR involved 
osteolysis after total hip arthroplasty by regulating FOXO1 expression to inhibit osteoblast differentiation. J Biomed Sci 2018;25:4.

29. Wang Y, Huo J, Zhang D, et al. Chemerin/ChemR23 axis triggers an inflammatory response in keratinocytes through ROS-sirt1-NF- $\kappa$ B signaling. J Cell Biochem 2019;120:6459-70.

30. van der Vorst EPC, Mandl M, Müller M, et al.
Hematopoietic ChemR23 (Chemerin Receptor 23) fuels atherosclerosis by sustaining an M1 macrophagephenotype and guidance of plasmacytoid dendritic cells to murine lesions-brief report. Arterioscler Thromb Vasc Biol 2019;39:685-93. Erratum in: Arterioscler Thromb Vasc Biol 2020;40:e274.

(English Language Editor: D. Fitzgerald)
Cite this article as: Zhao F, Cang D, Zhang J, Zheng L. Chemerin/ChemR23 signaling mediates the effects of ultrahigh molecular weight polyethylene wear particles on the balance between osteoblast and osteoclast differentiation. Ann Transl Med 2021;9(14):1149. doi: 10.21037/atm-21-2945 

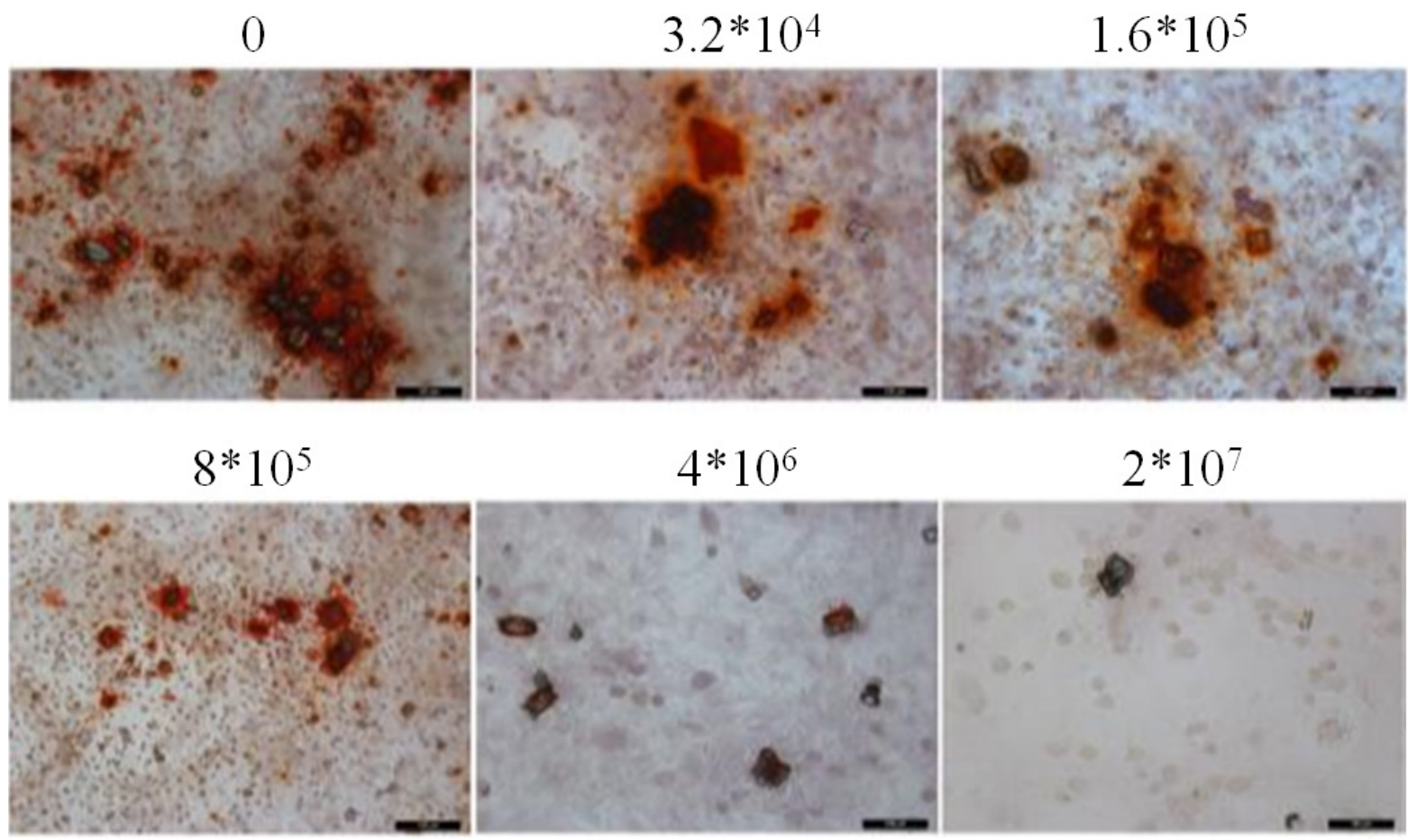

Figure S1 Osteoblast differentiation was inhibited by UHMWPE particles in a dose-dependent manner. MC3T3-E1 cells were cultured in osteoblast differentiation medium with different amount of UHMWPE particles for 21 days before being subjected to Alizarin red staining. Bar $=100 \mu \mathrm{m}$. 